\title{
Epidermoid spinal cord tumour after lumbar puncture
}

\author{
S J HALCROW, P J CRAWFORD, AND A W CRAFT \\ Departments of Neurosurgery, Newcastle General Hospital and Child Health, Royal Victoria Infirmary, \\ Newcastle upon Tyne
}

SUMMARY A 5 year old boy developed an implantation dermoid tumour after a lumbar puncture in infancy. A survey of senior paediatricians has shown it to be common practice to use an unstiletted needle for lumbar puncture in small children. A stiletted needle is recommended for all such procedures.

There is considerable evidence that epidermoid spinal cord tumours may be a late complication of lumbar puncture, particularly when it is carried out with a needle without a stylet. ${ }^{1}$ Because of this possibility it is recommended that lumbar puncture is always done with a stiletted needle. ${ }^{2}$ We report the occurrence of such a tumour in a 6 year old child who had had a lumbar puncture as an infant.

\section{Case report}

A chinese boy first presented at the age of 10 weeks with a short history of fever and vomiting. Meningitis was suspected and two unsuccessful lumbar punctures were performed. The type of needle used was not recorded. Subsequently pus cells were found in a specimen of urine and the urinary tract infection was treated with an antibiotic. An intravenous pyelogram was carried out and was normal.

He was well until 5 years and 9 months of age when he developed a pain in his left hip for which no cause was found. The pain persisted, however, and he also developed a dull ache in the lumbar region that radiated into the buttocks and was aggravated by coughing. Examination at this time showed a very stiff lumbar spine with straight leg raising limited to $45^{\circ}$ on both sides. There was a mild weakness of hip flexors and reduced ankle jerks. There was no disturbance of sphincter function. There was no clinical evidence of either spinal dysraphism or neurofibromatosis. Plain radiographs of the lumbar spine were normal. A myelogram was then performed, and this showed a mass extending from L3 to L4 and displacing nerve roots bilaterally around it. Cerebrospinal fluid was normal. A laminectomy of L3 and L4 was carried out and a 'pearly' mass was removed. The tumour measured
$30 \times 5 \mathrm{~mm}$ and was shown histologically to be a typical implantation dermoid. He made a complete recovery after the operation with no abnormal neurological signs, but he has developed noticeable keloid formation of the scar.

\section{Discussion}

Spinal cord tumours in children are uncommon, accounting for less than one fifth of all central nervous system tumours. ${ }^{3}$ Of those occurring in the spinal cord, only $3 \%$ are epidermoid in nature. ${ }^{4}$ Intraspinal epidermoid tumours in children are therefore rare. A search of the records of the regional neurological centre in Newcastle over the past 30 years showed only three other such tumours in the lumbar region, and all occurred in patients over the age of 30 years with no history of lumbar puncture. It may be that the reporting of this complication of lumbar puncture in the late 1960s has led to the abandonment of the practice of using a needle without a stilette. To test this hypothesis a simple questionnaire was sent to 30 senior paediatricians in this region. They were asked to report their current practice in newborns and in other small children, and 29 replied. Sixteen reported that they always used a stiletted needle for newborns, and 10 always used a 'butterfly' or ordinary needle. A further three stated that they varied. For other small children, 27 stated that they always used a stilette, and one that he always used an ordinary needle. The other varied. It would seem therefore, that although recommendations are that lumbar puncture should be carried out with a proper lumbar puncture needle ${ }^{2}$ there is widespread use of ordinary needles for this purpose. The reasons for this are probably that it is easier, particularly in small babies, to carry out a lumbar puncture with an ordinary needle, although it may also be that the hazards of such a procedure are not well recognised.

There are two theories on the causation of these tumours. Firstly, that they arise from embryonic rests of epidermal cells, or secondly that the practice of lumbar puncture without a stilette implants a few epidermal cells into the spinal canal and that these can grow later. The second is a more likely occurrence in children, as epidermoid tumours are 
very uncommonly reported in the absence of a lumbar puncture having been carried out.

The current practice of more intensive care of the newborn, with lumbar puncture being a not infrequent part of 'infection screens' carried out by junior staff, may lead to the occurrence of more implantation epidermoid tumours in the future. We strongly recommend the use of the smallest lumbar puncture needle available with the stilette in place until the skin has been punctured. The stilette can then be removed and the needle advanced until the dura is penetrated and cerebrospinal fluid obtained.
References

${ }^{1}$ Shaywitz BA. Epidermoid spinal cord tumours and previous lumbar puncture. J Pediatr 1972;80:638-40.

2 Lissauer T. Practical procedures in children (2). Hospital Update 1982:1545-53.

3 Ingraham FD, Matson DD. Neurosurgery of infancy and childhood. Springfield: Charles C Thomas, 1954.

${ }^{4}$ Rand RW, Rand CW. Intraspinal tumors of childhood. Springfield: Charles C Thomas, 1960.

Correspondence to Dr A W Craft, The Royal Victoria Infirmary, Queen Victoria Road, Newcastle upon Tyne NE1 4LP.

Received 18 June 1985

\title{
Obtaining parental consent-opting in or opting out?
}

\section{MUTCH AND R KING}

\author{
National Perinatal Epidemiology Unit and Oxford Region Child Development Project, Radcliffe \\ Infirmary, Oxford
}

SUMMARY In a population based project aimed at identifying children with specified disabilities within a health region, there was, rightly, concern about the transfer of data on named children across health district boundaries. Two methods of obtaining parental consent for this process were tested. High recruitment rates were achieved using an 'opting out' approach.

Throughout the health services much information about patients is collected, for several reasons. First and foremost, information is collected about specific individuals to assist in the diagnosis and management of their illnesses. Subsequent communication of this information between different arms of the health service requires the naming of the specific individuals.

Secondly, information is collected about groups of patients for the purpose of describing patterns of clinical practice, or for planning future health services. Such information does not need to identify specific individuals in order to be useful.

The provision of health services for children poses a particular problem. The emphasis is on preventive and screening measures for all children and information is required not just about those who are ill. The health services themselves must initiate the contact, obtaining information from the time of birth on all children identified by name, date of birth, and place of residence.

When screening programmes show potential problems and diagnostic referral is indicated, information passes from the preventive to the diagnostic and therapeutic services, without concern for confidentiality because it is considered to be in the ultimate best interest of the individual patient. Prior discussion, however, concerning the acceptability of the referral should have taken place with the parents.

The routine examinations of infants by health visitors has the tacit approval of the parents because they bring their child to a clinic or allow access to them at home.

In the Oxford Region Child Development Project, health visitors apply standardised screening procedures to all infants weighing less than $2000 \mathrm{~g}$, and to those who required special care in the neonatal period for more than 24 hours. The results are relayed centrally to the project office, together with the results of diagnostic referral, so that the number of individual children with specific disabilities in this geographically defined population can be documented. Because of our concern to acknowledge the confidential nature of this information, and because it involves transfer of information from the health services to a research project, it was important to seek the permission of parents for this transfer according to the principles set out in the report of the British Medical Association's Central Ethical Committee. 1

Anecdotal evidence (Alberman, Stanleypersonal communication) suggested that an 'opting out' approach to determine parental permission achieved a higher recruitment rate than 'opting in'. 